\title{
AN EXAMPLE CONCERNING BOUNDED LINEAR REGULARITY OF SUBSPACES IN HILBERT SPACE
}

\author{
SIMEON REICH ${ }^{凶}$ and ALEXANDER J. ZASLAVSKI
}

(Received 1 February 2013; accepted 10 July 2013; first published online 12 September 2013)

\begin{abstract}
We study bounded linear regularity of finite sets of closed subspaces in a Hilbert space. In particular, we construct for each natural number $n \geq 3$ a set of $n$ closed subspaces of $\ell^{2}$ which has the bounded linear regularity property, while the bounded linear regularity property does not hold for each one of its nonempty, proper nonsingleton subsets. We also establish a related theorem regarding the bounded regularity property in metric spaces.
\end{abstract}

2010 Mathematics subject classification: primary 46C05.

Keywords and phrases: bounded regularity, Hilbert space, subspace.

\section{Introduction}

Infinite products of (linear and nonlinear) operators and, in particular, infinite products of retractions are often used for approximating points in the intersection of a finite number $m \geq 2$ of closed and convex sets via individual (best) approximations from each one of these sets. If all the sets are closed linear subspaces $S_{1}, S_{2}, \ldots, S_{m}$ (or, more generally, closed affine subspaces), of a given (real) Hilbert space $H$, then the best approximations to a given point $x \in H$ in each one of the subspaces $S_{i}$ coincide with the orthogonal projections $P_{S_{i}} x$. Taking an arbitrary $x_{0} \in H$ and defining the iterations $x_{n}=\left(P_{S_{m}} P_{S_{m-1}} \cdots P_{S_{1}}\right)^{n} x_{0}, n=1,2, \ldots$, we obtain a sequence $\left\{x_{n}\right\}$ which converges in norm to an element $x^{*} \in S:=S_{1} \cap \cdots \cap S_{m}$. This fundamental fact was first established for $m=2$ by von Neumann in [3] and for the general case by Halperin in [2].

The theorems in $[2,3]$ did not state any rate of the above-mentioned convergence and in fact, it is now known that this convergence may be arbitrarily slow. Any uniform estimate of the convergence rate that was proved since then in some particular cases required special interplay between the subspaces $S_{i}$, for example, positivity of the angles between them and some of their intersections (see, for example, [4]). The properties of a given set of subspaces $\left\{S_{1}, S_{2}, \ldots, S_{m}\right\}$ guaranteeing uniform

This research was supported by the Israel Science Foundation (Grant No. 647/07), the Fund for the Promotion of Research at the Technion and by the Technion VPR Research Fund.

(C) 2013 Australian Mathematical Publishing Association Inc. 0004-9727/2013 \$16.00 
convergence of infinite products were investigated by Bauschke and Borwein in [1], where they proved that this kind of convergence occurred if and only if the subspace $S_{1}^{\perp}+S_{2}^{\perp}+\cdots+S_{m}^{\perp}$ is closed in $H$. This property was named bounded linear regularity of the given set of subspaces.

To provide a quantitative character to the property of regularity, one can use the inclination of a given set of subspaces $\left\{S_{1}, S_{2}, \ldots, S_{m}\right\}$, where $m \geq 2$, which is defined by

$$
\ell\left(S_{1}, S_{2}, \ldots, S_{m}\right)=\inf _{x \notin S} \frac{\max _{1 \leq j \leq m}\left\|x-P_{S_{j}} x\right\|}{\left\|x-P_{S} x\right\|} .
$$

Thus we see that the inclination compares the distances from an arbitrary point $x \in H$ to the subspaces $S_{i}$ and to their intersection. When the inclination is positive, not only one obtains the uniform convergence of the infinite products, but also an estimate of the rate of this convergence. Many useful applications of the concept of inclination can be found in [5]. As a matter of fact, the positivity of the inclination (and of the inner inclination [6]) is equivalent to the above-mentioned bounded regularity property.

In the next section we exhibit for any natural number $n \geq 3$, a set $A=$ $\left\{X_{1}, X_{2}, \ldots, X_{n}\right\}$ of $n$ closed linear subspaces of $\ell^{2}$ with the following properties:

(i) the bounded linear regularity property does not hold for each nonempty, proper nonsingleton subset of $A$ (that is, its inclination is zero);

(ii) the whole set $A$ has the bounded linear regularity property (that is, its inclination is positive).

So we see that the bounded linear regularity property is not hereditary.

Property (i) is easily deduced from Proposition 2.1 below, while property (ii) follows from Proposition 2.2.

In Section 3 we turn our attention to the bounded regularity property in general metric spaces. We show (see Theorem 3.1 below) that if for each $j \in\{1, \ldots, q\}$, $\mathcal{L}_{j}=\left\{C_{j, i}: i=1, \ldots, n_{j}\right\}$ is a finite collection of nonempty subsets of a metric space $X$ such that $\bigcap_{j=1}^{q}\left(\cap_{i=1}^{n_{j}} C_{j, i}\right) \neq \emptyset$ and $\mathcal{L}_{j}$ has the bounded regularity property for each $j \in\{1, \ldots, q\}$, then the family

$$
\mathcal{L}=\left\{C_{j, i}: j \in\{1, \ldots, q\}, i \in\left\{1, \ldots, n_{j}\right\}\right\}
$$

possesses the bounded regularity property if and only if the family

$$
\tilde{\mathcal{L}}=\left\{\bigcap_{i=1}^{n_{j}} C_{j, i}: j=1, \ldots, q\right\}
$$

has this property.

\section{Bounded linear regularity of subspaces}

Denote by $X=\ell^{2}$ the Hilbert space consisting of all the sequences of real numbers $\left\{x_{i}\right\}_{i=1}^{\infty}$ such that $\sum_{i=1}^{\infty} x_{i}^{2}<\infty$, with the inner product

$$
\langle x, y\rangle=\sum_{i=1}^{\infty} x_{i} y_{i}, \quad x=\left\{x_{i}\right\}_{i=1}^{\infty}, y=\left\{y_{i}\right\}_{i=1}^{\infty} \in X
$$


This inner product induces the norm

$$
\|x\|=\langle x, x\rangle^{1 / 2}=\left(\sum_{i=1}^{\infty} x_{i}^{2}\right)^{1 / 2}, \quad x=\left\{x_{i}\right\}_{i=1}^{\infty} \in X .
$$

Let $D_{1}, D_{2} \subset X$ be nonempty sets. We say that $D_{1}$ and $D_{2}$ are orthogonal and write $D_{1} \perp D_{2}$ if $\langle u, v\rangle=0$ for all $u \in D_{1}$ and $v \in D_{2}$.

Let $n \geq 3$ be a natural number and let $e_{i}, i=1,2, \ldots$, be the standard basis in $X$. Namely, for $j=1,2, \ldots, e_{j}=\left\{e_{j, i}\right\}_{i=1}^{\infty}$ with

$$
e_{j, j}=1, \quad e_{j, i}=0 \quad \text { for all integers } i \geq 1 \text { such that } i \neq j .
$$

For each $x \in X$ and each nonempty set $D \subset X$, set $\rho(x, D)=\inf \{\|x-y\|: y \in D\}$. Denote by $L_{1}$ the closed linear subspace generated by the vectors $\left\{e_{2 i+1}: i=1,2, \ldots\right\}$ and by $L_{2}$ the closed linear subspace generated by $\left\{e_{2 i}: i=1,2, \ldots\right\}$.

For each pair of nonempty sets $D_{1}, D_{2} \subset X$, set

$$
D_{1}+D_{2}=\left\{x+y: x \in D_{1}, y \in D_{2}\right\} \text {. }
$$

Let $L$ and $\tilde{L}$ be two closed linear subspaces of $L_{1}$ such that

$$
L+\tilde{L}=L_{1} \quad \text { and } \quad L \perp \tilde{L} .
$$

As we have already mentioned, we are going to construct a set $A=\left\{X_{1}, \ldots, X_{n}\right\}$ of $n \geq 3$ closed linear subspaces of $\ell^{2}$ with properties (i) and (ii). We construct them in such a way that the following property also holds:

(iii) $X_{j_{1}} \cap X_{j_{2}}=L$ for all $j_{1}, j_{2} \in\{1, \ldots, n\}$ such that $j_{1} \neq j_{2}$.

Since $L$ is an arbitrary linear closed subspace of $L_{1}$, we can actually obtain many examples of sets $A$ with properties (i)-(iii).

To this end, we first construct closed linear subspaces $X_{0, s} \subset L_{2}, s=1, \ldots, n$, such that $X_{s_{1}} \cap X_{s_{2}}=\{0\}$ for all $s_{1}, s_{2} \in\{1, \ldots, n\}$ satisfying $s_{1} \neq s_{2}$ and then for all $s=1, \ldots, n$, we set $X_{s}=X_{0, s}+L$. Since $L_{2} \perp L$, we conclude that property (iii) holds.

For each natural number $k$, each $j \in\{1, \ldots, n\}$ and each $i \in\{1, \ldots, n-1\}$, set

$$
\xi_{(n-1) n(k-1)+(j-1)(n-1)+i}=e_{2 n^{2}(k-1)+2(j-1) n+2}+2^{-k} e_{2 n^{2}(k-1)+2(j-1) n+2(i+1)} .
$$

Clearly, we have defined $\xi_{i}, i=1,2, \ldots$.

Let $s \in\{1, \ldots, n\}$. Denote by $X_{0, s}$ the closed linear subspace generated by the basis vectors

$\left\{\xi_{(n-1) n(k-1)+(j-1)(n-1)+s-1}: k\right.$ is a natural number, $j \in\{1, \ldots, n\}$ and $\left.j<s\right\}$

$\cup\left\{\xi_{(n-1) n(k-1)+(j-1)(n-1)+s}: k\right.$ is a natural number, $j \in\{1, \ldots, n\}$ and $\left.j>s\right\}$.

By (2.2) and (2.3),

$$
X_{0, s} \perp L_{1}
$$


By (2.2), if $k_{1}$ and $k_{2}$ are natural numbers such that $j_{1}, j_{2} \in\{1, \ldots, n\}, i_{1}, i_{2} \in$ $\{1, \ldots, n-1\}$ and $\left(k_{1}, j_{1}\right) \neq\left(k_{2}, j_{2}\right)$, then

$$
\left\langle\xi_{(n-1) n\left(k_{1}-1\right)+\left(j_{1}-1\right)(n-1)+i_{1}}, \xi_{(n-1) n\left(k_{2}-1\right)+\left(j_{2}-1\right)(n-1)+i_{2}}\right\rangle=0 .
$$

By (2.2), the system of vectors $\xi_{i}, i=1,2, \ldots$, is linearly independent. When combined with (2.3), this implies that for all $s_{1}, s_{2} \in\{1, \ldots, n\}$ satisfying $s_{1} \neq s_{2}$,

$$
X_{0, s_{1}} \cap X_{0, s_{2}}=\{0\} .
$$

Now for each $s \in\{1, \ldots, n\}$, set

$$
X_{s}=X_{0, s}+L
$$

By (2.1) and (2.4), $X_{s}$ is closed for all $s \in\{1, \ldots, n\}$.

Our first proposition shows that the set $A=\left\{X_{1}, X_{2}, \ldots, X_{n}\right\}$ has property (i).

Proposition 2.1. Let $\epsilon \in(0,1)$ and $j \in\{1, \ldots, n\}$. Then there is a point $z \in L_{2}$ such that $\|z\|=\rho(z, L)=1$ and $\rho\left(z, X_{i}\right) \leq \epsilon$ for all $i \in\{1, \ldots, n\} \backslash\{j\}$.

Proof. Choose a natural number $k$ such that

$$
2^{-k}<\epsilon / 2
$$

There are two cases: $j=1$ and $j>1$.

Assume first that $j=1$. Then by (2.3) and (2.6),

$$
\xi_{(n-1) n(k-1)+s-1} \in X_{0, s} \subset X_{s}, \quad s=2, \ldots, n .
$$

Let

$$
z=e_{2 n^{2}(k-1)+2} .
$$

By (2.3) and (2.7)-(2.9), for all $s \in\{1, \ldots, n\} \backslash\{1\}$,

$$
\rho\left(z, X_{s}\right) \leq\left\|z-\xi_{(n-1) n(k-1)+s-1}\right\|=\left\|e_{2 n^{2}(k-1)+2}-\xi_{(n-1) n(k-1)+s-1}\right\| \leq 2^{-k}<\epsilon / 2
$$

and the assertion of the proposition follows.

Assume next that $j>1$. Then by (2.3) and (2.6), for all $s=1, \ldots, j-1$,

$$
\xi_{(n-1) n(k-1)+(j-1)(n-1)+s} \in X_{0, s} \subset X_{s}
$$

and for all integers $s$ satisfying $j<s \leq n$,

$$
\xi_{(n-1) n(k-1)+(j-1)(n-1)+s-1} \in X_{0, s} \subset X_{s} .
$$

Define

$$
z=e_{2 n^{2}(k-1)+2(j-1) n+2} .
$$


By (2.9)-(2.12), (2.2) and (2.7), for all $s \in\{1, \ldots, n\} \backslash\{j\}$,

$$
\begin{aligned}
& \rho\left(z, X_{s}\right) \leq \rho\left(z, X_{0, s}\right)=\rho\left(e_{2 n^{2}(k-1)+2+2(j-1) n}, X_{0, s}\right) \\
& \quad \leq \max \left\{\left\|e_{2 n^{2}(k-1)+2(j-1) n+2}-\xi_{(n-1) n(k-1)+(j-1)(n-1)+i}\right\|: i=1, \ldots, n-1\right\} \\
& \quad \leq 2^{-k}<\epsilon / 2
\end{aligned}
$$

and the assertion of the proposition follows once again.

This completes the proof of Proposition 2.1.

Next we show that the set $A$ has property (ii).

Proposition 2.2. Let $x \in X$ with

$$
\rho\left(x, \bigcap_{j=1}^{n} X_{j}\right) \geq 1 .
$$

Then there is $p \in\{1, \ldots, n\}$ such that $\rho\left(x, X_{p}\right) \geq(2+2 n)^{-1}$.

By (2.1) and (2.4)-(2.6),

$$
\bigcap_{j=1}^{n} X_{j}=L .
$$

Clearly, there is a vector $y \in X$ such that

$$
y \in L, \quad\|x-y\|=\inf \{\|x-u\|: u \in L\}, \quad\{x-y\} \perp L .
$$

There also is a point $z \in X$ such that

$$
z \in \tilde{L}, \quad\|x-y-z\|=\inf \{\|x-y-u\|: u \in \tilde{L}\}, \quad\{x-y-z\} \perp \tilde{L} .
$$

By (2.1), (2.15) and (2.16),

$$
\{x-y-z\} \perp L_{1}
$$

By (2.13)-(2.15),

$$
\|x-y\| \geq 1
$$

There are two cases:

$$
\|z\|>2^{-1} \text { and } \quad\|z\| \leq 2^{-1} .
$$

Assume that

$$
\|z\|>2^{-1}
$$

Let $j \in\{1, \ldots, n\}$. By (2.16), (2.6), (2.1), (2.4) and the definition of $L_{1}$,

$$
\{z\} \perp X_{j}
$$

Let

$$
u \in X_{j}
$$


By (2.19) and (2.20),

$$
\langle u, z\rangle=0 .
$$

By (2.16) and (2.21),

$$
\langle x-y-z-u, z\rangle=0 .
$$

It follows from (2.22) that

$$
\|x-y-u\|^{2}=\|(x-y-z-u)+z\|^{2}=\|x-y-z-u\|^{2}+\|z\|^{2} \geq\|z\|^{2}>4^{-1} .
$$

Since $u$ is an arbitrary element of $X_{j}$, we conclude that $\rho\left(x-y, X_{j}\right) \geq 2^{-1}$. Since $y \in L \subset X_{j}$ (see (2.6) and (2.15)), this implies that $\rho\left(x, X_{j}\right) \geq 2^{-1}$ for all $j \in\{1, \ldots, n\}$ and the assertion of the proposition follows.

Assume now that

$$
\|z\| \leq 2^{-1}
$$

By (2.17) and (2.23),

$$
\|x-y-z\| \geq 2^{-1}
$$

By (2.1), (2.16) and (2.15),

$$
\{x-y-z\} \perp L_{1}
$$

Thus

$$
x-y-z \in L_{2} .
$$

For any $j \in\{1, \ldots, n\}$, denote by $Y_{j}$ the closed linear subspace generated by the vectors

$$
\left\{\xi_{(n-1) n(k-1)+(j-1)(n-1)+i}: k \text { is a natural number, } i \in\{1, \ldots, n-1\}\right\} .
$$

By the definition of $X_{j}, Y_{j}, j=1, \ldots, n$, and $\xi_{i}, i=1,2, \ldots$, we have, for any $j \in$ $\{1, \ldots, n\}$,

$$
Y_{j} \subset L_{2}, \quad Y_{j} \perp L_{1}, \quad Y_{j} \perp X_{j}, \quad Y_{j_{1}} \perp Y_{j_{2}}
$$

for all $j_{1}, j_{2} \in\{1, \ldots, n\}$ such that $j_{1} \neq j_{2}$.

Clearly, $Y_{1}+Y_{2}+\cdots+Y_{n}$ is a closed subspace of $L_{2}$. Set

$$
Y_{0}=\left\{y \in L_{2}:\{y\} \perp Y_{i}, i=1, \ldots, n\right\} .
$$

Clearly, $Y_{0}$ is a closed linear subspace of $L_{2}$ (it may happen that $Y_{0}=\{0\}$ ) and

$$
Y_{0}+Y_{1}+\cdots+Y_{n}=L_{2}
$$

By (2.25) and (2.28), there exist points

$$
y_{i} \in Y_{i}, \quad i=0, \ldots, n,
$$

such that

$$
\sum_{i=0}^{n} y_{i}=x-y-z
$$


By definition,

$$
X_{0,1}+X_{0,2}+\cdots+X_{0, n} \subset Y_{1}+Y_{2}+\cdots+Y_{n}
$$

By (2.24) and (2.30), there is an integer $j \in\{0, \ldots, n\}$ such that

$$
\left\|y_{j}\right\| \geq 2^{-1}(n+1)^{-1} \text {. }
$$

By (2.6), (2.16), (2.1), (2.4), (2.29) and (2.27),

$$
\left\{z+y_{0}\right\} \perp X_{i}, \quad i=1, \ldots, n .
$$

There are two cases: $j=0$ and $j \neq 0$.

Assume first that $j=0$. Let

$$
i \in\{1, \ldots, n\} \quad \text { and } \quad u \in X_{i} .
$$

By (2.31)-(2.33), (2.16), (2.1), (2.25), (2.29), (2.28), (2.30), (2.27) and (2.18),

$$
\begin{aligned}
\|x-y-u\|^{2}= & \left\|x-y-z-y_{0}-u+z+y_{0}\right\|^{2} \\
\geq & \left\|z+y_{0}\right\|^{2}+\left\|x-y-z-y_{0}-u\right\|^{2} \\
& \quad+2\left\langle z+y_{0}, x-y-z-y_{0}-u\right\rangle \\
\geq & \left\|z+y_{0}\right\|^{2}+2\left\langle z+y_{0}, x-y-z-y_{0}\right\rangle \\
= & \left\|z+y_{0}\right\|^{2}+2\left\langle y_{0}, x-y-z-y_{0}\right\rangle \\
= & \left\|z+y_{0}\right\|^{2}=\|z\|^{2}+\left\|y_{0}\right\|^{2} \geq 4^{-1}(n+1)^{-2}
\end{aligned}
$$

and

$$
\begin{gathered}
\|x-y-u\| \geq 2^{-1}(n+1)^{-1} \quad \text { for all } u \in X_{i}, \\
\rho\left(x-y, X_{i}\right) \geq 2^{-1}(n+1)^{-1} .
\end{gathered}
$$

Since $y \in L($ see $(2.15))$,

$$
\rho\left(x, X_{i}\right) \geq 2^{-1}(n+1)^{-1} \text { for all } i=1, \ldots, n
$$

and the assertion of the proposition follows.

Assume now that $j \neq 0$. Let

$$
u \in X_{j}
$$

Clearly,

$$
\begin{aligned}
\|x-y-u\|^{2} & =\left\|x-y-z-y_{j}-u+z+y_{j}\right\|^{2} \\
& \geq\left\|z+y_{j}\right\|^{2}+2\left\langle z+y_{j}, x-y-z-y_{j}-u\right\rangle \\
& \geq\left\|z+y_{j}\right\|^{2}+2\left\langle z+y_{j}, x-y-z-y_{j}\right\rangle-2\left\langle z+y_{j}, u\right\rangle .
\end{aligned}
$$

By (2.16), (2.34), (2.6), (2.1), (2.2), (2.29) and (2.26),

$$
\left\langle z+y_{j}, u\right\rangle=\langle z, u\rangle+\left\langle y_{j}, u\right\rangle=0 .
$$


By (2.16), (2.28), (2.30), (2.26), (2.27) and (2.28),

$$
\left\langle z+y_{j}, x-y-z-y_{j}\right\rangle=\left\langle y_{j}, x-y-z-y_{j}\right\rangle=0 .
$$

By (2.35), (2.36), (2.37), (2.16), (2.29), (2.28), (2.1), (2.31) and (2.32),

$$
\begin{gathered}
\|x-y-u\|^{2} \geq\left\|z+y_{j}\right\|^{2}=\|z\|^{2}+\left\|y_{j}\right\|^{2} \geq 4^{-1}(n+1)^{-2}, \\
\|x-y-u\| \geq 2^{-1}(n+1)^{-1} \quad \text { for all } u \in X_{j}
\end{gathered}
$$

and

$$
\rho\left(x-y, X_{j}\right) \geq 2^{-1}(n+1)^{-1} .
$$

Since $y \in L \subset X_{j}$ (see (2.6) and (2.15)), $\rho\left(x, X_{j}\right) \geq 2^{-1}(n+1)^{-1}$ and the assertion of the proposition follows once again.

This completes the proof of Proposition 2.2.

\section{Bounded regularity in metric spaces}

In this section we state and prove a theorem regarding bounded regularity in general metric spaces.

Let $(X, \rho)$ be a metric space. For each point $x \in X$ and each subset $A \subset X$, set $\rho(x, A)=\inf \{\rho(x, y): y \in A\}$. Fix a point $\theta \in X$.

Let $\mathcal{L}=\left\{X_{1}, \ldots, X_{n}\right\}$ be a finite collection of nonempty subsets of $X$ such that $\bigcap_{i=1}^{n} X_{i} \neq \emptyset$.

Recall that $\mathcal{L}$ is said to have the bounded regularity property [1] if for each $\epsilon>0$ and $M>0$, there exists $\delta>0$ such that:

(P1) if $x \in X$ satisfies $\rho(x, \theta) \leq M$ and $\rho\left(x, X_{i}\right)<\delta, i=1, \ldots, n$, then

$$
\rho\left(x, \bigcap_{i=1}^{n} X_{i}\right)<\epsilon .
$$

Suppose that $\mathcal{L}$ possesses the bounded regularity property. Let $\epsilon>0$ and $M>0$ be given. Denote by $\mathcal{D}(M, \epsilon)$ the set of all numbers $\delta \in(0, \epsilon]$ for which (P1) holds and set

$$
\phi_{\mathcal{L}}(\epsilon, M)=\sup (\mathcal{D}(\epsilon, M)) .
$$

It is clear that $\phi_{\mathcal{L}}(\epsilon, M) \leq \epsilon$ and (P1) holds with $\delta=\phi_{\mathcal{L}}(\epsilon, M)$.

Theorem 3.1. Suppose that $q$ is a natural number. For each integer $j \in\{1, \ldots, q\}$, let $\mathcal{L}_{j}=\left\{C_{j, i}: i=1, \ldots, n_{j}\right\}$ be a finite collection of nonempty subsets of $X$ such that $\bigcap_{i=1}^{n_{j}} C_{j, i} \neq \emptyset, \mathcal{L}_{j}$ possesses the bounded regularity property and

$$
\bigcap_{j=1}^{q}\left(\bigcap_{i=1}^{n_{j}} C_{j, i}\right) \neq \emptyset .
$$

Let

$$
\mathcal{L}=\left\{C_{j, i}: j \in\{1, \ldots, q\}, i \in\left\{1, \ldots, n_{j}\right\}\right\}
$$


and

$$
\tilde{\mathcal{L}}=\left\{\bigcap_{i=1}^{n_{j}} C_{j, i}: j=1, \ldots, q\right\} .
$$

Then $\mathcal{L}$ has the bounded regularity property if and only if $\tilde{\mathcal{L}}$ has the bounded regularity property. If they possess the bounded regularity property, then for each $\epsilon, M>0$,

$$
\phi_{\tilde{\mathcal{L}}}(\epsilon, M) \geq \phi_{\mathcal{L}}(\epsilon, M) \geq \min \left\{\phi_{\mathcal{L}_{j}}\left(\phi_{\tilde{\mathcal{L}}}(\epsilon, M), M\right): j=1, \ldots, q\right\} .
$$

Proof. Assume that $\mathcal{L}$ possesses the bounded regularity property. To show that $\tilde{\mathcal{L}}$ possesses the bounded regularity property, let $\epsilon$ and $M$ be positive. Let $x \in X$, $\rho(x, \theta) \leq M$,

$$
\rho\left(x, \bigcap_{i=1}^{n_{j}} C_{j, i}\right)<\phi_{\mathcal{L}}(\epsilon, M), \quad j=1, \ldots, q .
$$

By (3.1), the bounded regularity property of $\mathcal{L}$ and the definition of $\phi_{\mathcal{L}}$,

$$
\rho\left(x, C_{j, i}\right)<\phi_{\mathcal{L}}(\epsilon, M), \quad j=1, \ldots, q, i=1, \ldots, n_{j},
$$

and $\epsilon>\rho\left(x, \bigcap_{j=1}^{q}\left(\bigcap_{i=1}^{n_{j}} C_{j, i}\right)\right)$. Thus $\tilde{\mathcal{L}}$ has the bounded regularity property and $\phi_{\tilde{\mathcal{L}}}(\epsilon, M) \geq \phi_{\mathcal{L}}(\epsilon, M)$.

In the other direction, assume that $\tilde{\mathcal{L}}$ has the bounded regularity property. To show that $\mathcal{L}$ possesses the bounded regularity property, let $\epsilon$ and $M$ be positive. Let $x \in X, \rho(x, \theta) \leq M$,

$\rho\left(x, C_{j, i}\right)<\min \left\{\phi_{\mathcal{L}_{j}}\left(\phi_{\tilde{L}}(\epsilon, M), M\right): j=1, \ldots, q\right\}, \quad j=1, \ldots, q, i=1, \ldots, n_{j}$.

Let $j \in\{1, \ldots, q\}$. By (3.2) and the bounded regularity of $\mathcal{L}_{j}$,

$$
\rho\left(x, \bigcap_{i=1}^{n_{j}} C_{j, i}\right)<\phi_{\tilde{\mathcal{L}}}(\epsilon, M) .
$$

By (3.2), (3.3) and the bounded regularity of $\tilde{\mathcal{L}}$,

$$
\rho\left(x, \bigcap_{j=1}^{q}\left(\bigcap_{i=1}^{n_{j}} C_{j, i}\right)\right)<\epsilon .
$$

Thus $\mathcal{L}$ possesses the bounded regularity property and

$$
\phi_{\mathcal{L}}(\epsilon, M) \geq \min \left\{\phi_{\mathcal{L}_{j}}\left(\phi_{\tilde{L}}(\epsilon, M), M\right): j=1, \ldots, q\right\} .
$$

This completes the proof of Theorem 3.1. 


\section{References}

[1] H. H. Bauschke and J. M. Borwein, 'On projection algorithms for solving convex feasibility problems', SIAM Rev. 38 (1996), 367-426.

[2] I. Halperin, 'The product of projection operators', Acta Sci. Math. (Szeged) 23 (1962), 96-99.

[3] J. von Neumann, 'On rings of operators. Reduction theory', Ann. of Math. (2) 50 (1949), 401-485.

[4] E. Pustylnik, S. Reich and A. J. Zaslavski, 'Convergence of non-cyclic infinite products of operators', J. Math. Anal. Appl. 380 (2011), 759-767.

[5] E. Pustylnik, S. Reich and A. J. Zaslavski, 'Convergence of non-periodic infinite products of orthogonal projections and nonexpansive operators in Hilbert space', J. Approx. Theory 164 (2012), 611-624.

[6] E. Pustylnik, S. Reich and A. J. Zaslavski, 'Inner inclination of subspaces and infinite products of orthogonal projections', J. Nonlinear Convex Anal. 14 (2013), 423-436.

SIMEON REICH, Department of Mathematics,

The Technion - Israel Institute of Technology, 32000 Haifa, Israel

e-mail: sreich@tx.technion.ac.il

ALEXANDER J. ZASLAVSKI, Department of Mathematics, The Technion - Israel Institute of Technology, 32000 Haifa, Israel e-mail: ajzasl@tx.technion.ac.il 